\title{
Brain peak width of skeletonised mean diffusivity (PSMD), processing speed, and other cognitive domains
}

Ian J. Deary ${ }^{a, b, c, *}$, Stuart J. Ritchie ${ }^{a, b}$, Susana Muñoz Maniega ${ }^{b, d, e}$, Simon R. Cox, ${ }^{a, b, e}$ Maria C.

Valdés Hernández ${ }^{b, d, e, f}$, John M. Starr ${ }^{b, c}$, Joanna M. Wardlaw ${ }^{b, d, e, f}$, Mark E. Bastin ${ }^{b, d, e}$

${ }^{a}$ Department of Psychology, University of Edinburgh, Edinburgh, United Kingdom EH8 9JZ

${ }^{\mathrm{b}}$ Centre for Cognitive Ageing and Cognitive Epidemiology, University of Edinburgh,

Edinburgh, United Kingdom EH8 9JZ

'Alzheimer Scotland Dementia Research Centre, University of Edinburgh, Edinburgh, United Kingdom EH8 9JZ

${ }^{d}$ Brain Research Imaging Centre, Division of Neuroimaging Sciences, University of Edinburgh, Edinburgh, United Kingdom EH16 4SB

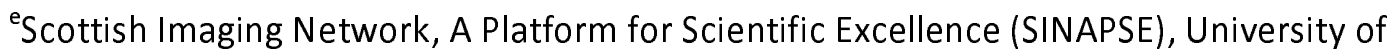
Edinburgh, Edinburgh, United Kingdom EH16 4SB

fEdinburgh Dementia Research Centre, Dementia Research Institute, University of Edinburgh, Edinburgh, United Kingdom EH16 4SB

*Corresponding author at: Department of Psychology, University of Edinburgh, 7 George

Square, Edinburgh, EH8 9JZ, United Kingdom. Tel.: +44 1316503452.

E-mail address: i.deary@ed.ac.uk (I.J. Deary). 


\begin{abstract}
It is suggested that the brain's peak width of skeletonised water mean diffusivity (PSMD) is a neuro-biomarker of processing speed, a crucial contributor to cognitive ageing. We tested whether PSMD is more strongly correlated with processing speed than with other cognitive domains, and more strongly than other structural brain MRI indices. Participants were 731 Lothian Birth Cohort 1936 members, mean age 73 years (SD=0.7); analytical sample was 656-680. Cognitive domains tested were: processing speed (5 tests), visuospatial (3), memory (3), and verbal (3). Brain-imaging variables included PSMD, white matter diffusion parameters and hyperintensity volumes, grey and white matter volumes, and perivascular spaces. PSMD was significantly associated with all processing speed tests; absolute standardised beta values were 0.11 to 0.23 (mean $=0.17$ ). Other structural brain-imaging variables correlated as or more strongly. PSMD was significantly associated with processing speed (-0.27), visuospatial (-0.23), memory (-0.17), and general cognitive ability $(-0.25)$. PSMD correlated with processing speed: but not more strongly than with other cognitive domains; and not more strongly than other brain-imaging measures.
\end{abstract}

Keywords: ageing, cognition, processing speed, structural MRI, diffusion MRI, white matter, PSMD 


\section{Introduction}

Cognitive functions such as processing speed, reasoning, and some aspects of memory decline, on average, as people grow older, with deleterious effects on people's quality of life (Tucker-Drob, 2011; Salthouse, 2017; Institute of Medicine, 2015). Processing speed has a special place among the cognitive domains; it has been suggested as a foundation for longstanding differences in, and ageing of, other cognitive domains (Salthouse, 1996, 2000; Deary, 2000; Verhaeghen, 2014; Ritchie et al., 2014). With regard to understanding cognitive ageing, detailed attention has been advocated in the study of the psychometric aspects of processing speed and its potential neurobiological correlates (Salthouse and Madden, 2008). Therefore, understanding the neurobiological foundations of people's differences in, and ageing of, processing speed and other cognitive domains is a research priority (Penke et al., 2012; Salthouse, 2000).

Even among those with no overt disease, brain imaging-derived biomarkers of brain deterioration correlate with performance on cognitive tests; such markers include cerebral tissue volumes and brain atrophy, and indicators of white matter health and so-called cerebral small vessel disease (Arvanitakis et al., 2016; Cremers et al., 2016; Dong et al., 2015; Gazes et al., 2016; Liu et al., 2017; Salthouse et al., 2015). Cerebral small vessel disease is an important contributor to vascular-based cognitive deterioration (Shi and Wardlaw, 2016). Recognising that, a brain imaging marker named 'peak width of skeletonized [water] mean diffusivity' (PSMD) was developed and studied with respect to its association with processing speed (Baykara et al., 2016). It is based on skeletonization and histogram analysis of diffusion tensor magnetic resonance imaging (DT-MRI) data. PSMD was examined in comparison with other brain imaging markers in patients with inherited 
and sporadic cerebral small vessel disease, and in patients with Alzheimer's disease and healthy controls (Baykara et al., 2016). PSMD was reported to be associated with processing speed in all samples; the authors reported that it correlated with speed more strongly than other brain imaging markers such as brain volume, volume of white matter hyperintensities, and volume of lacunes.

However, although a special association between PSMD and the cognitive domain of processing speed was emphasised by Baykara et al. (2016), the test that was used to examine processing speed in most samples-the Trail Making Test-is not specific to that domain; Trail Making Test performance is associated with general fluid cognitive ability as well as processing speed (Salthouse, 2011; MacPherson et al., 2017). Moreover, no other cognitive domains were examined by Baykara et al. (2016) for their associations with PSMD. It is therefore not clear whether PSMD correlates with purer processing speed tests, or with processing speed more than it does with other cognitive domains. In addition, there were no formal comparisons undertaken by Baykara et al. (2016) between PSMD's and other brain imaging biomarkers' associations with 'processing speed' (mostly Trail Making Test performance), nor with other cognitive domains. This means that there was no formal test of whether PSMD correlated better with processing speed-or with other cognitive domains-more strongly than with other structural brain imaging biomarkers. Such a series of tests is necessary before concluding that PSMD is a special neuro-biomarker of the important cognitive domain of processing speed. As Schmidt (2017) explained, all cognitive domains correlate positively together, and, therefore, associations with any one domain might represent an association with general cognitive function rather than a domain-specific relation. 
To test whether PSMD has a privileged association with processing speed has the following desiderata, all of which are met in the present study. First, there should be appropriate, relatively-specific tests of processing speed, to test their associations with PSMD. Second, there should be a variety of other cognitive measures, to enable tests of the relation of PSMD to processing speed in comparison with other cognitive domains. Third, PSMD should be compared with other structural brain imaging biomarkers to test whether its association with processing speed and other cognitive domains is especially strong. Here, we run these analyses in a narrow-age sample of mostly healthy, community-dwelling older people: the Lothian Birth Cohort 1936 (LBC1936).

\section{Materials and Methods}

\subsection{Participants}

All participants were members of the LBC1936 (Deary et al., 2007, 2012; Taylor et al., in press). This started (LBC1936, Wave 1) as a sample of 1,091 people who were recruited between 2004 and 2007 at about 70 years of age. All lived independently in the community, were generally healthy, and travelled to a clinical research facility for assessment. Most of them had taken part in the Scottish Mental Survey 1947 at age 11 years. They undertook extensive cognitive, medical, biomarker, psycho-social and other assessments. The assessments were repeated at Wave 2 , about three years later at mean age 73 years, with the addition of a detailed structural brain MRI scan (Wardlaw et al., 2011). Of the 886 who returned at Wave 2 , over 700 agreed to undertake a brain scan. All variables described below were collected at Wave 2, unless otherwise noted. Ethical approval for the LBC1936 study came from the Multi-Centre Research Ethics Committee for Scotland (MREC/01/0/56; 
07/MRE00/58) and the Lothian Research Ethics Committee (LREC/2003/2/29). All

participants, who were volunteers and received no financial or other reward, completed a written consent form before any testing took place.

\subsection{Cognitive tests}

The cognitive test data used here are from Wave 2 , at mean age 73 years. All participants had already taken the same cognitive test battery at age 70 years, ensuring that they were familiar with the tests.

2.2.1 Processing speed. Because processing speed was the key cognitive variable mentioned in relation with PSMD (Baykara et al., 2016), it is apposite that the LBC1936 has been assessed for processing speed in detail. There were five tests of processing speed: two were paper-and-pencil psychometric tests; two were experimental, reaction time tests; and one was a psychophysical test. The psychometric tests were from the Wechsler Adult Intelligence Test-III ${ }^{\mathrm{UK}}$ : Digit Symbol and Symbol Search (Wechsler, 1998a). The reaction time tests were simple reaction time ( 8 practice trials, 20 test trials), and 4-choice reaction time (8 practice trials, 40 test trials), assessed on a stand-alone device that was used in the UK's Health and Lifestyle Study (Deary et al., 2001). The psychophysical test was inspection time, assessed using a bespoke computer program (Deary et al., 2004). The inspection time task required the participant to indicate which of two briefly-presented parallel vertical lines was longer; no speeded response was required, and only the correctness of the response was recorded. Stimuli were backward-masked. There were 150 trials, with ten trials at each of 15 durations, ranging from $6 \mathrm{~ms}$ to $200 \mathrm{~ms}$. Durations were presented at random, using a method of constant stimuli. 
2.2.2 Visuospatial ability. This was assessed using Matrix Reasoning and Block Design from the Wechsler Adult Intelligence Test-III ${ }^{\mathrm{UK}}$ (Wechsler, 1998a), and Spatial Span (total score of forward and backward) from the Wechsler Memory Scales-III ${ }^{\mathrm{UK}}$ (Wechsler, 1998b).

2.2.3 Verbal memory. This was assessed using Verbal Paired Associates (total score) and Logical Memory (immediate and delayed total score) from the Wechsler Memory Scales-III ${ }^{\mathrm{UK}}$ (Wechsler, 1998b) and Backward Digit Span from the Wechsler Adult Intelligence Test-III ${ }^{\mathrm{UK}}$ (Wechsler, 1998a).

2.2.4 Crystallised ability. This was assessed using the National Adult Reading Test (Nelson and Willison 1991), the Wechsler Test of Adult Reading (Holdnack, 2001), and a phonemic verbal fluency test (using the letters C, F, and L; Deary et al., 2007).

\subsection{Demographic and health-related variables}

The participant's own occupational social class was based on their most prestigious job prior to retirement, and assessed on a 6-point scale from manual to professional (Office of Population and Census Studies, 1980). Education was assessed as the number of years of full time education. These were assessed at Wave 1. Smoking was assessed at interview and classed as never, ex-, or current. The Mini-Mental State Examination (Folstein et al., 1975) was used as a screen for cognitive pathology, and not used as part of the cognitive test battery. Participants reported if they had a history of hypertension, cardiovascular disease, or diabetes. 


\subsection{Magnetic resonance imaging}

All MRI data were acquired using a GE Signa Horizon HDxt 1.5 T clinical scanner (General Electric, Milwaukee, WI, USA) using a self-shielding gradient set with maximum gradient strength of $33 \mathrm{mT} / \mathrm{m}$ and an 8-channel phased-array head coil. The full details of the imaging protocol can be found in the LBC1936 imaging protocol paper (Wardlaw et al., 2011). Briefly, the DT-MRI examination consisted of $7 T_{2}$-weighted $\left(b=0 \mathrm{~s} \mathrm{~mm}^{-2}\right.$ ) and sets of diffusion-weighted $\left(b=1000 \mathrm{~s} \mathrm{~mm}^{-2}\right)$ single-shot spin-echo echo-planar (EP) volumes acquired with diffusion gradients applied in 64 non-collinear directions (Jones et al., 2002). Volumes were acquired in the axial plane, with a field-of-view of $256 \times 256 \mathrm{~mm}$, contiguous slice locations, and image matrix and slice thickness designed to give $2 \mathrm{~mm}$ isotropic voxels. The repetition and echo time for each EP volume were $16.5 \mathrm{~s}$ and $98 \mathrm{~ms}$ respectively. DTMRI data were converted from DICOM (http://dicom.nema.org) to NIfTI-1 (http://nifti.nimh.nih.gov/nifti-1) format using the TractoR package (http://www.tractor-mri.org.uk) (Clayden at al., 2011). FSL tools (http://www.fmrib.ox.ac.uk/fsl) (Smith at al., 2004) were then used to extract the brain, remove bulk motion and eddy current induced distortions by registering all subsequent volumes to the first $\mathrm{T}_{2}$-weighted EP volume (Jenkinson and Smith, 2001), estimate the water diffusion tensor and calculate parametric maps of mean diffusivity (MD) and fractional anisotropy (FA) from its eigenvalues using DTIFIT (Basser and Pierpaoli, 1996).

\subsubsection{Peak width of skeletonized water mean diffusivity. Automatic calculation of PSMD}

followed the procedure described by Baykara et al. (2016) using the freely-available script they provided (http://www.psmd-marker.com). Briefly, the DT-MRI data were processed using the standard Tract-based Spatial Statistics (TBSS; Smith at al., 2006) pipeline available 
in FSL with histogram analysis performed on the resulting white matter MD skeletons. First, all participants' FA volumes were linearly and non-linearly registered to the standard space FMRIB 1 mm FA template. Second, a white matter skeleton was created from the mean of all registered FA volumes. This was achieved by searching for maximum FA values in directions perpendicular to the local tract direction in the mean FA volume. An FA threshold of 0.2 was applied to the mean FA skeleton to exclude predominantly non-white matter voxels. Third, MD volumes were projected onto the mean FA skeleton and further thresholded at an FA value of 0.3 to reduce CSF partial volume contamination. Finally, PSMD was calculated as the difference between the $95^{\text {th }}$ and $5^{\text {th }}$ percentiles of the voxel-based MD values within each subject's MD skeleton.

2.4.2 Other structural brain imaging variables. The estimation of, respectively, normalappearing grey and white matter volumes, brain atrophy (intra-cranial volume minus total brain volume), general FA and general MD derived from quantitative tractography, white matter hyperintensity (WMH) volume, and visually-rated perivascular spaces (Potter et al., 2015) from LBC1936 Wave 2 have all been described previously, as, mostly, have their associations with cognitive functions (Royle et al., 2013; Valdes Hernandez at al., 2013; Penke et al., 2012; Booth et al., 2013; Ritchie et al., 2015a,b,c; Aribisala et al., in submission). They are included in the present paper in order to compare their cognitive associations alongside those of PSMD, not as new results in themselves.

\subsection{Statistical analysis}

All analyses were run in the lavaan package for R (Rosseel, 2012). Scores on each multi-test cognitive domain (processing speed, visuospatial ability, verbal memory, and crystallised 
ability) were derived from separate confirmatory factor analyses assessed using structural equation models based on the analytic sample. Scores on general cognitive ability were derived from a hierarchical confirmatory factor analysis where the score on the general cognitive factor represented the shared variance among the four cognitive domains. Associations between brain imaging parameters and cognitive test scores were conducted using linear regression, with p-values adjusted for the False Discovery Rate (FDR; Benjamini and Hochberg, 1995) as indicated in results tables. To test whether the relation of each cognitive test or domain with PSMD was significantly different from its relation with the other brain-derived variables, we used Williams's test for differences in dependent correlations (implemented using the psych package in $\mathrm{R}$ (Revelle, 2018); treating the standardised regression betas as correlations for this purpose. For each test, we accounted for the dependency (correlation) between the brain variables.

As an additional multivariate analysis, we ran a set of structural equation models in which all eight of the brain measures were entered simultaneously as predictors of the latent cognitive scores. This allowed us to test whether PSMD was incrementally significant beyond the more conventionally-studied micro- and macro-structural brain measures. For the general FA and MD factors, we extracted the factor scores to use as predictors, but still estimated the cognitive factors as latent variables. As with the previous analyses, all variables were corrected for sex and age at the time of testing/scanning. Note that we already reported a similar analysis in this sample (Ritchie et al., 2015b). However, the previous analysis did not include PSMD, and its aim was to discover how much of the variance in cognitive domains could be accounted for with structural brain variables. In the present analysis the aim was to test whether PSMD had an incremental contribution in 
predicting cognitive variation, in a situation where several structural brain variables were included simultaneously.

\section{Results}

Summary demographic, medical, brain imaging, and cognitive results are shown in Table 1.

The total number of subjects who agreed to brain imaging was 731 (388 men), and between 656 and 680 of them provided brain imaging data that were able to be used to compute variables for use in this study. Their mean age was 72.7 years $(S D=0.72)$. About $47 \%$ had a history of hypertension, $27 \%$ cardiovascular disease, and $10 \%$ diabetes. Their mean MiniMental State Examination score was $28.8(S D=1.4)$.

The age- and sex-adjusted correlations between the brain imaging measures used in this study are shown in Supplementary Table 1. PSMD correlated-in terms of absolute standardized effect sizes-above 0.5 with general FA and MD, and WMH volume, about 0.3 with atrophy and perivascular spaces, and below 0.2 with grey and white matter volumes.

For clarity in understanding the results reported below, all significant associations were in the direction indicating that healthier brains had better performance in processing speed and other cognitive domains. Healthier brains had lower PSMD, higher general FA, lower general MD, lower WMH volume, higher grey and white matter volumes, lower atrophy, and fewer perivascular spaces.

3.1 PSMD and other brain imaging variables versus processing speed. We first examined the associations between the five tests of processing speed and PSMD and the other brain 
imaging measures (Table 2). Higher PSMD (representing less healthy white matter) was significantly associated with worse scores on all five measures of processing speed; the absolute standardised betas were between 0.11 and 0.23 (mean $=0.17$ ). Those with higher PSMD had lower scores on Digit Symbol, Symbol Search, and inspection time, and slower simple and 4-choice reaction times. Therefore, PSMD does correlate significantly, in the expected direction, with these five methodologically-varied tests of processing speed.

Normal-appearing grey and white matter volumes, and brain atrophy had similar associations to PSMD with the five processing speed measures (Table 2); 11 of their 15 betas were larger in effect size (though not significantly larger) than those of PSMD. Associations between WMH volume and the processing speed tests were similar to those of PSMD, though mostly slightly lower. General FA and MD had significant associations with all five of the processing speed measures, with similar betas to those between PSMD and speed measures, though the majority were slightly lower. Perivascular spaces had non-significant associations with the five processing speed measures, and all were notably lower than those with PSMD. We repeated these analyses, omitting participants with a Mini-Mental State Examination score below 24; the results were very similar (Supplementary Table 2).

We tested the effect sizes of the associations between PSMD and individual processing speed tests to find out formally whether they were significantly stronger or weaker than those between other brain imaging variables and the same test. Apart from perivascular spaces, where PSMD always had stronger associations with processing speed measures, PSMD had significantly stronger associations than other brain variables in only one of 30 comparisons (Table 2). 


\subsection{PSMD and other brain imaging variables' associations with four cognitive domains and}

general cognitive ability. Next, we examined the possibility that PSMD showed significantly

stronger associations with processing speed than with other cognitive domains, and

whether they were significantly stronger or weaker associations compared to other brain

imaging variables. We ascertained the associations between PSMD and other brain imaging

measures and the four cognitive domains (each of which comprised multiple cognitive tests)

and general cognitive ability (the variance shared by the four cognitive domains) (Table 3).

Higher PSMD was significantly associated, at similar effect sizes, with poorer processing

speed (standardised beta $=-0.27)$, visuospatial ability $(-0.23)$, and general cognitive ability $(-$

$0.25)$. The association with verbal memory was significant but lower $(-0.17)$, and the

association with crystallised ability was small and non-significant (-0.07).

For all five of the cognitive variables, normal-appearing grey and white matter volumes and brain atrophy had stronger associations than PSMD (Table 3). The associations of WMH

volume were somewhat lower than those of PSMD, and all but the association with

crystallised ability were significant. General FA and MD had significant associations with all cognitive domains, except between MD and crystallised ability, and the betas were mostly slightly lower than those between PSMD and cognitive domains. Perivascular spaces had no significant associations with any cognitive domain.

We repeated these analyses, omitting participants with a Mini-Mental State Examination score below 24; the results were very similar (Supplementary Table 3). Additional analyses show the associations between the various structural brain imaging measures and the 
individual tests from the cognitive domains of Visuospatial ability, Memory, and Crystallised ability (Supplementary Tables 4, 5, and 6, respectively).

We tested the effect sizes of the associations between PSMD and each cognitive domain and general cognitive ability to find out formally whether they were significantly stronger or weaker than those between other brain imaging variables and the same cognitive variable. Table 3 shows these results. There were 35 comparisons: in 11 of these, PSMD had stronger associations with cognitive domains and general cognitive ability; and in five of these the PSMD associations were significantly weaker. For processing speed, the cognitive domain of principal interest here, PSMD was a significantly stronger associate than general MD and perivascular spaces, and significantly weaker than white matter volume. Therefore, although PSMD does correlate significantly, in the expected direction, with these cognitive measures, it did not exhibit the largest association among all brain measures in any cognitive domain.

\subsection{Multivariate models to test PSMD's incremental contribution to predicting variance in} cognitive domains and general cognitive ability. The results of the multivariate models, where all brain variables were entered together to predict variance in cognitive domain scores and general cognitive ability, are shown in Table 4. In these models, PSMD had a statistically significant association with visuospatial ability and with the general cognitive ability factor, but not with processing speed, verbal memory, or crystallized ability. No one brain measure consistently emerged as a statistically significant predictor for each cognitive ability; we note that grey matter volume, white matter volume, and atrophy were all independently significant alongside PSMD in predicting general cognitive ability. The general 
FA and MD variables were not independently significant in any of the models, indicating that variation in the cognitive abilities was better measured by either the macrostructural indices (except perivascular spaces, which were also not significant in any model), or by PSMD.

\section{Discussion}

In this large sample of community-dwelling older people with a narrow age range around 73 years, we attempted a replication of the previously-reported links between PSMD and processing speed (Baykara et al., 2016). Importantly, we extended the analysis to include necessary comparisons with a variety of more specific processing speed tests, other cognitive domains, and other brain measures. Consistent with the results of the first PSMDcognitive function report (Baykara et al., 2016), higher PSMD correlated significantly with poorer performance on five methodologically-diverse tests of processing speed: two were psychometric, paper and pencil tests; two were experimental, reaction time tests; and one was a psychophysical test, requiring no fast motor response. Higher PSMD correlated significantly with poorer performance on the latent cognitive domain of processing speed, composed of tests of all three types. However, PSMD was not the exclusive or strongest associate of processing speed: normal-appearing grey and white matter volumes and brain atrophy were often slightly stronger, and $\mathrm{WMH}$ volume and tractography-based general FA and MD had slightly lower, but still-significant associations. Moreover, PSMD did not have an exclusive or especially strong association with processing speed compared with other cognitive domains; it correlated at similar levels with visuospatial ability and general cognitive ability, though less strongly with verbal memory and crystallised ability. In multivariate analyses, PSMD contributed predictive power, incremental to that of other 
structural brain imaging variables, to visuospatial ability and general cognitive ability, but not to processing speed.

If PSMD's association with processing speed had been higher than those of other structural brain indices, and/or if PSMD has been especially strong in associating with the domain of processing speed by comparison with other cognitive domains, then there would have been strong evidence for arguing that PSMD stands out among brain imaging parameters in being especially associated with this important cognitive domain. Because all cognitive domains are quite strongly correlated, it is important always to test other domains before making a claim that a putative biomarker or any other variable has a special association with one of them; quite often, a variable is, in fact, associated with general cognitive function, which means that it will be associated with most individual cognitive domains. This pitfall (i.e. incorrectly claiming variable $\mathrm{X}$ has a particular association with cognitive domain $\mathrm{Y}$, in cases where only domain $\mathrm{Y}$ was tested) is common and has been discussed at length (Schmidt, 2017).

Nevertheless, the present study's results might still be evidence of an important and special association between PSMD and processing speed. Although PSMD did no better than other brain imaging parameters in associating with processing speed, and was also associated with other cognitive domains, it might be argued that, because of its ease of computation and tractability, it is superior to measures that are burdensome to compute and which are more general properties of the brain. However, global measures of GM and WM volume can be obtained from a T1 alone, and their automated measurement can also be undertaken in an automated fashion. That is, both PSMD's convenience and link to more specific brain 
biology might be used to argue in its favour; the average magnitude of water molecular diffusion in the centre of common white matter pathways provides information on individual variations in white matter microstructure and the specific methods reduce the likelihood of influences of partial volume effects, rather than simply 'how much' of a tissue type an individual possesses. Moreover, the similar association of PSMD with three cognitive domains might not detract from the claim that it is a marker of processing speed. This is because processing speed has long been seen as somewhat special among the cognitive domains. It has been argued that faster processing speed might provide a foundation for better cognitive performance in higher cognitive domains and for less cognitive decline in them (Salthouse, 1996; Verhaeghen, 2014; Ritchie et al., 2014). This is because the tests of processing speed are arguably simpler than those of other cognitive domains, often being referred to as elementary cognitive tests. Thus, if links to underlying biological processes are clearer for PSMD compared with other structural brain markers, and for processing speed compared with other cognitive domains, then it may be argued PSMD is primus inter (apparent) pares for the brain indices examined here. Furthermore, code for performing PSMD is freely available and based on the commonly-used TBSS pipeline making it an accessible biomarker of white matter structure.

The study has the strengths of testing a large and age- and culturally-homogeneous sample, all on the same brain scanner. Processing speed was tested thoroughly, at three levels of description (psychometric, experimental, and psychophysical). The other main cognitive domains that show age-related decline-visuospatial reasoning and memory-and crystallised ability were assessed using multiple, well-validated tests. Also, recognising that all cognitive domains correlate strongly, a general cognitive ability variable, capturing their 
shared variance, was included. The study has some limitations. The homogeneity of age and cultural background mean that we are not able to generalise beyond those. Neither can we generalise beyond their status as relatively-healthy, community-dwelling individuals; it is possible that samples with a broader range of brain pathology might show stronger brain imaging-cognitive domain associations, and possibly a pattern that brings out more of PSMD's distinctiveness. The original PSMD-validation study (Baykara et al., 2016) had the advantage of multiple clinical and healthy groups, but its limitations included that most of its samples did not have a relatively-specific test of processing speed and there were insufficient tests of other cognitive domains.

The present study assessed the claim that PSMD is associated with processing speed. We found the predicted association, but it was not exclusive or special with respect to other structural brain indices, or with respect to other cognitive domains. However, PSMD's specificity, and processing speed's possible special status among cognitive domains, make the PSMD-processing speed association worth exploring and explaining in a wider range of clinical and non-clinical populations.

\section{Acknowledgements}

The work was undertaken as part of the Cross Council and University of Edinburgh Centre for Cognitive Ageing and Cognitive Epidemiology (CCACE; http://www.ccace.ed.ac.uk). This work was supported by a Research into Ageing programme grant (to I.J.D. and J.M.S.) and the Age UK-funded Disconnected Mind project (http://www.disconnectedmind.ed.ac.uk; to I.J.D., J.M.S. and J.M.W.), with additional funding from the UK Medical Research Council (MRC; to I.J.D., J.M.S., J.M.W., M.E.B., S.J.R. and S.R.C.). J.M.W. is supported by the Scottish 
Funding Council through the SINAPSE Collaboration (http://www.sinapse.ac.uk). CCACE

(MRC MR/K026992/1) is funded by the Biotechnology and Biological Sciences Research

Council and MRC. The image acquisition and analysis was performed at the Brain Research Imaging Centre, University of Edinburgh (http://www.bric.ed.ac.uk). The authors thank the

research team members who collected, processed, collated and checked cognitive, medical, and brain imaging data. We thank Dr Natalie Royle for her work on some of the non-PSMD brain imaging variables used in the present study.

\section{Disclosure statement}

The authors declare no competing financial interests.

\section{References}

Aribisala, BS, Riha R, Valdes Hernandez M, Maniega SM, Cox S, Radakovic R, Taylor A, Pattie

A, Corley J, Redmond P, Bastin M, Starr J, Deary IJ, Wardlaw JM. The association of sleep duration and quality with brain perivascular spaces, white matter hyperintensities and brain atrophy. In submission.

Arvanitakis Z, Fleischman DA, Arfanakis K, Leurgans SE, Barnes LL, Bennett DA. Association of white matter hyperintensities and grey matter volume with cognition in older individuals without cognitive impairment. Brain Struct Funct 2016;221:2135-2146.

Basser PJ, Pierpaoli C. Microstructural and physiological features of tissues elucidated by quantitative-diffusion-tensor MRI. J Magn Reson 1996;292:155-170. 
Baykara E, Gesierich B, Adam R, Tuladhar, AM, Biesbroek JM, Koek HL, Ropele S, Jouvent E, Alzheimer's Disease Neuroimaging Initiative, Chabriat H, Ertl-Wagner B, Ewers M, Schmidt R, de Leeuw FE, Biessels GJ, Dichgans M, Duering M. A novel imaging marker for small vessel disease based on skeletonization of white matter tracts and diffusion histograms. Ann Neurol 2016;80:581-592.

Benjamini Y, Hochberg Y. Controlling the false discover rate: a practical and powerful approach to multiple testing. J R Stat Soc Series B Stat Methodol 1995;57:289-300.

Booth T, Bastin ME, Penke L, Maniega SM, Murray C, Royle NA, Gow AJ, Corley J, Henderson RD, Hernandez MC, Starr JM, Wardlaw JM, Deary IJ. Brain white matter tract integrity and cognitive abilities in community-dwelling older people: the Lothian Birth Cohort 1936.

Neuropsychology 2013;27:595-607.

Clayden JD, Maniega, SM, Storkey AJ, King MD, Bastin ME, Clark CA. TractoR: magnetic resonance imaging and tractography with R. J Stat Softw 2011;44:1-18.

Cremers LGM, de Groot M, Hofman A, Krestin GP, van der Lugt A, Niessen WJ, Vernooij MW, Ikram MA. Altered tract-specific white matter microstructure is related to poorer cognitive performance: The Rotterdam Study. Neurobiol Aging 2016;39:108-117.

Deary IJ. Looking down on human intelligence; from psychometric to the brain. Oxford, UK: Oxford University Press; 2000. 
Deary IJ, Der G, Ford G. Reaction times and intelligence differences: a population-based cohort study. Intelligence 2001;29:389-399.

Deary IJ, Gow AJ, Taylor MD, Corley J, Brett C, Wilson V, Campbell H, Whalley U, Visscher PM, Porteous DJ, Starr JM. The Lothian Birth Cohort 1936: a study to examine influences on cognitive ageing from age 11 to age 70 and beyond. BMC Geriatr 2007;7:28.

Deary IJ, Gow AJ, Pattie A, Starr JM. Cohort profile: the Lothian Birth Cohorts of 1921 and 1936. Int J Epidemiol 2012;41:1576-1584.

Deary IJ, Simonotto E, Meyer M, Marshall A, Marshall I, Goddard N, Wardlaw JM. The functional anatomy of inspection time: an event-related fMRI study. Neurolmage 2004;22:1466-1479.

Dong C, Nabizadeh N, Caunca M, Cheung YK, Rundek T, Elkind MSV, DeCarli C, Sacco RL, Stern Y, Wright CB. Cognitive correlates of white matter lesion load and brain atrophy. Neurology 2015;85:441-449.

Folstein MF, Folstein SE, McHugh PR. Mini-Mental State: A practical method for grading the cognitive state of patients for the clinician. J Psychiatr Res 1975;12:189-198.

Gazes Y, Bowman FD, Razlighi QR, O’Shea D, Stern Y, Habeck C. White matter tract covariance patterns predict age-declining cognitive abilities. Neurolmage 2016;125:53-60. 
Holdnack JA. WTAR: Wechsler Test of Adult Reading manual. San Antonio, TX: Psychological Corporation; 2001.

Institute of Medicine. Cognitive aging: Process in understanding and opportunities for action. Washington, DC: The National Academies Press; 2015.

Jenkinson M, Smith S. A global optimisation method for robust affine registration of brain images. Med Imag Anal 2001;5:143-156.

Jones DK, Williams SC, Gasston D, Horsfield MA, Simmons A, Howard R. Isotopic resolution diffusion tensor imaging with whole brain acquisition in a clinically acceptable time. Hum Brain Mapp 2002;15:216-230.

Liu H, Yang Y, Xia Y, Zhu W, Leak RK, Wei Z, Wang J, Hu X. Aging of cerebral white matter. Ageing Res Rev 2017;34:64-76.

MacPherson SE, Cox SR, Dickie DA, Karama S, Starr JM, Evans AC, Bastin ME, Wardlaw JM, Deary IJ. Processing speed and the relationship between Trail Making Test-B performance, cortical thinning and white matter microstructure in older adults. Cortex 2017;95:92-13.

Nelson HE, Willison JR. National Adult Reading Test (NART) Test Manual (Part II). Windsor, UK: NFER-Nelson; 1991. 
Office of Population and Census Surveys. Classification of occupations. London, UK: Her Majesty's Stationery Office; 1980.

Penke L, Munoz Maniega A, Bastin ME, Valdes Hernandez MC, Murray C, Royle NA, Starr JM, Wardlaw JM, Deary IJ. Brain white matter tract integrity as a neural foundation for general intelligence. Mol Psychiatry 2012;17:1026-1030.

Potter GM, Chappell FM, Morris Z, Wardlaw JM. Cerebral perivascular spaces visible on magnetic resonance imaging: Development of a qualitative rating scale and its observer reliability. Cerebrovasc Dis 2015;39:224-231

Revelle, W. psych: Procedures for Personality and Psychological Research, Northwestern University, Evanston, Illinois, USA; 2018.

Ritchie SJ, Bastin ME, Tucker-Drob EM, Maniega SM, Engelhardt LE, Cox SR, Royle NA, Gow AJ, Corley J, Pattie A, Taylor AM, Valdes Hernandez MC, Starr JM, Wardlaw JM, Deary IJ. Coupled changes in brain white matter microstructure and fluid intelligence in later life. J Neurosci 2015a;35:8672-8682.

Ritchie SJ, Booth T, Valdes Hernandez M, Corley J, Maniega SM, Gow AJ, Royle NA, Pattie A, Karama S, Starr JM, Bastin ME, Wardlaw JM, Deary IJ. Beyond a bigger brain: multivariable structural brain imaging and intelligence. Intelligence 2015b;51:47-56. 
Ritchie SJ, Dickie DA, Cox SR, Valdes Hernandez MC, Corley J, Royle NA, Pattie A, Aribisala B,

Redmond P, Maniega SM, Taylor AM, Sibbett, R, Gow AJ, Starr JM, Bastin ME, Wardlaw JM,

Deary IJ. Brain volumetric changes and cognitive ageing during the eighth decade of life.

Hum Brain Mapp 2015c;36:4910-4925.

Ritchie SJ, Tucker-Drob EM, Deary IJ. A strong link between speed of visual discrimination and cognitive ageing. Curr Biol 2014;24:R681-683.

Rosseel Y. An R package for structural equation modelling. J Stat Softw 2012;48:1-36.

Royle NA, Booth T, Valdes Hernandez MC, Penke L, Murray C, Gow AJ, Maniega SM, Starr J, Bastin ME, Deary IJ, Wardlaw JM. Estimated maximal and current brain volume predict cognitive ability in old age. Neurobiol Aging 2013;34:2726-2733.

Salthouse TA. The processing-speed theory of adult age differences in cognition. Psychol Rev 1996;103:403-428.

Salthouse TA. Aging and measures of processing speed. Biol Psychol 2000;54:35-54.

Salthouse TA. What cognitive abilities are involved in trail-making performance? Intelligence 2011;39:222-232.

Salthouse TA. Contributions of the individual differences approach to cognitive ageing. J Gerontol B Psychol Sci Soc Sci 2017;72:7-15. 
Salthouse TA, Habeck C, Razlighi Q, Barulli D, Gazes Y, Stern Y. Breadth and age-dependency of relations between cortical thickness and cognition. Neurobiol Aging 2015;36:3020-3028.

Salthouse TA, Madden DJ. Information processing speed and aging. In: DeLuca J, Kalmar JH, editors. Information processing speed in clinical populations. New York: Taylor and Francis; 2008, p. 221-242.

Schmidt FL. Beyond questionable research methods: the role of omitted relevant research in the credibility of research. Arch Sci Psychol 2017;5:32-41.

Shi Y, Wardlaw JM. Update on cerebral small vessel disease: a dynamic whole-brain disease. Stroke Vasc Neurol 2016;1:83-92.

Smith SM, Jenkinson M, Johansen-Berg H, Rueckert D, Nichols TE, Mackay CE, Watkins KE, Ciccarelli O, Cader MZ, Matthews PM, Behrens TE. Tract-based spatial statistics: voxelwise analysis of multi-subject diffusion data. Neurolmage 2006;31:1487-1505.

Smith SM, Jenkinson M, Woolrich MW, Beckmann CF, Behrens TE, Johansen-Berg H, Bannister PR, De Luca M, Drobnjak I, Flitney DE, Niazy RK, Saunders J, Vickers J, Zhang Y, De Stefano N, Brady JM, Matthews PM. Advances in functional and structural MR image analysis and implementation as FSL. Neurolmage 2004;23:S208-S219. 
Taylor A, Pattie A, Deary IJ. Cohort profile update: The Lothian Birth Cohorts of 1921 and 1936. Int J Epidemiol, in press.

Tucker-Drob EM. Global and domain-specific changes in cognition throughout adulthood.

Dev Psychol 2011;47:331-343.

Valdes Hernandez MC, Booth T, Murray C, Gow AJ, Penke L, Morris Z, Maniega SM, Royle

NA, Aribisala BS, Bastin ME, Starr JM, Deary IJ, Wardlaw JM. Brain white matter damage in aging and cognitive ability in youth and older age. Neurobiol Aging 2013;34:2740-2747.

Verhaeghen P. The Elements of Cognitive Ageing; Meta-analysis of Age-related Differences in Processing Speed and Their Consequences. Oxford, UK: Oxford University Press; 2014.

Wardlaw JM, Bastin ME, Valdes Hernandez MC, Maniega SM, Royle NA, Morris Z, Clayden

JD, Sandeman EM, Eadie E, Murray C, Starr JM, Deary IJ. Brain ageing, cognition in youth and old age, and vascular disease in the Lothian Birth Cohort 1936: rationale, design and methodology of the imaging protocol. Int J Stroke 2011;6:547-559.

Wechsler D. WAIS-IIIUK administration and scoring manual. London, UK: Psychological Corporation; 1998a.

Wechsler D. WMS-IIIUK administration and scoring manual. London, UK: Psychological Corporation; 1998b. 
bioRxiv preprint doi: https://doi.org/10.1101/385013; this version posted August 4, 2018. The copyright holder for this preprint (which was not certified by peer review) is the author/funder, who has granted bioRxiv a license to display the preprint in perpetuity. It is made available under aCC-BY 4.0 International license. 
Table 1

Characteristics of the Lothian Birth Cohort 1936 study sample. Numbers are mean and SD or N and $\%$.

\begin{tabular}{|c|c|c|c|}
\hline Variable category & Variable & $\begin{array}{l}\text { Mean (SD) or N } \\
(\%)\end{array}$ & Total $\mathrm{N}$ \\
\hline \multirow[t]{6}{*}{ Demographic characteristics } & Age (years) & $72.68(0.72)$ & 866 \\
\hline & Sex; male, female & 388,343 & 1,091 \\
\hline & Adult social class & $3.49(1.21)$ & 1,091 \\
\hline & Education (years) & $10.79(1.14)$ & 1,091 \\
\hline & Smoking (never, ex-, current) & $343,327,61$ & 866 \\
\hline & $\begin{array}{l}\text { Mini-Mental State } \\
\text { examination }\end{array}$ & $28.76(1.41)$ & 865 \\
\hline \multirow[t]{4}{*}{ Medical conditions } & Hypertension (\%) & $362(46.9 \%)$ & 866 \\
\hline & Cardiovascular disease (\%) & $198(27.1 \%)$ & 866 \\
\hline & Diabetes (\%) & $77(10.5 \%)$ & 866 \\
\hline & Hypercholesterolemia (\%) & $356(41 . \%)$ & 866 \\
\hline \multirow[t]{7}{*}{$\begin{array}{l}\text { Structural brain imaging } \\
\text { measures }\end{array}$} & PSMD & $\begin{array}{l}3.17 \times 10^{-4} \\
\left(5.01 \times 10^{-5}\right) \\
\end{array}$ & 672 \\
\hline & General FA & $0.00(1.00)$ & 664 \\
\hline & General MD & $0.00(1.00)$ & 664 \\
\hline & WMH volume $\left(\mathrm{cm}^{3}\right)$ & $12.23(12.18)$ & 656 \\
\hline & Grey matter volume $\left(\mathrm{cm}^{3}\right)$ & $472.43(44.68)$ & 657 \\
\hline & White matter volume $\left(\mathrm{cm}^{3}\right)$ & $476.89(50.55)$ & 657 \\
\hline & Perivascular spaces & $3.51(1.10)$ & 680 \\
\hline \multirow[t]{5}{*}{ Processing speed tests } & Wechsler Digit Symbol & $56.29(12.42)$ & 862 \\
\hline & Wechsler Symbol Search & $24.62(6.19)$ & 862 \\
\hline & Simple reaction time $(\mathrm{s})$ & $0.27(0.05)$ & 865 \\
\hline & 4-choice reaction time $(\mathrm{s})$ & $0.65(0.09)$ & 865 \\
\hline & Inspection time & $111.37(11.75)$ & 838 \\
\hline \multirow[t]{3}{*}{ Visuospatial ability } & Wechsler Matrix Reasoning & $13.36(4.92)$ & 863 \\
\hline & Wechsler Block Design & $34.00(10.07)$ & 864 \\
\hline & Wechsler Spatial Span & $14.75(2.77)$ & 861 \\
\hline \multirow[t]{3}{*}{ Verbal memory } & $\begin{array}{l}\text { Wechsler Verbal Paired } \\
\text { Associates }\end{array}$ & $27.18(9.57)$ & 843 \\
\hline & Wechsler Logical Memory & $74.53(17.92)$ & 864 \\
\hline & $\begin{array}{l}\text { Wechsler Digit Span } \\
\text { Backward }\end{array}$ & $7.86(2.32)$ & 866 \\
\hline \multirow[t]{3}{*}{ Crystallised ability } & National Adult Reading Test & $34.36(8.16)$ & 864 \\
\hline & $\begin{array}{l}\text { Wechsler Test of Adult } \\
\text { Reading }\end{array}$ & $41.01(6.96)$ & 864 \\
\hline & Phonemic verbal fluency & $43.22(12.95)$ & 865 \\
\hline
\end{tabular}

Note: General FA and MD were latent variables created using full-information maximum-likelihood estimation from 12 white matter tract measurements; the total $\mathrm{N}$ given is the highest possible $\mathrm{n}$. 
Table 2

The association between structural brain imaging parameters and individual tests of processing speed.

\begin{tabular}{|c|c|c|c|c|c|c|c|c|c|c|c|c|c|c|c|}
\hline & \multicolumn{3}{|c|}{ Wechsler Digit Symbol } & \multicolumn{3}{|c|}{ Wechsler Symbol Search } & \multicolumn{3}{|c|}{ Simple reaction time } & \multicolumn{3}{|c|}{ 4-choice reaction time } & \multicolumn{3}{|c|}{ Inspection time } \\
\hline & 8 & SE & $p_{\text {adj }}$ & 8 & SE & $p_{\text {adj }}$ & 8 & SE & $p_{\text {adj }}$ & 8 & SE & $p_{\text {adj }}$ & 8 & SE & $p_{\text {adj }}$ \\
\hline PSMD & -.226 & .037 & $1.01 \times 10^{-08}$ & -.155 & .038 & $5.74 \times 10^{-05}$ & .108 & .037 & .003 & .169 & .037 & $1.04 \times 10^{-05}$ & -.200 & .037 & $3.02 \times 10^{-07}$ \\
\hline General FA & .188 & .041 & $1.70 \times 10^{-05}$ & .119 & .042 & .005 & -.147 & .044 & .001 & -.189 & .042 & $1.70 \times 10^{-05}$ & .172 & .042 & $8.14 \times 10^{-05}$ \\
\hline General MD & $-.143^{*}$ & .041 & .003 & -.092 & .042 & .028 & .121 & .043 & .008 & .113 & .043 & .010 & -.162 & .042 & $5.05 \times 10^{-04}$ \\
\hline WMH volume & -.200 & .037 & $5.55 \times 10^{-07}$ & -.164 & .038 & $3.13 \times 10^{-05}$ & .044 & .037 & .234 & .140 & .037 & $2.12 \times 10^{-04}$ & -.172 & .039 & $2.45 \times 10^{-05}$ \\
\hline Grey matter volume & .259 & .037 & $2.62 \times 10^{-11}$ & $.257 \dagger$ & .037 & $3.60 \times 10^{-11}$ & -.047 & .037 & .204 & -.164 & .037 & $1.48 \times 10^{-05}$ & .147 & .039 & $2.05 \times 10^{-04}$ \\
\hline White matter volume & .292 & .036 & $2.55 \times 10^{-14}$ & $.257+$ & .037 & $3.30 \times 10^{-11}$ & -.119 & .037 & .001 & -.215 & .036 & $7.45 \times 10^{-09}$ & .174 & .039 & $1.07 \times 10^{-05}$ \\
\hline Atrophy & .268 & .037 & $4.05 \times 10^{-12}$ & .215 & .038 & $3.16 \times 10^{-08}$ & -.109 & .037 & .003 & -.180 & .037 & $1.42 \times 10^{-06}$ & .220 & .038 & $3.16 \times 10^{-08}$ \\
\hline Perivascular spaces & $-.033^{*}$ & .038 & 0.646 & $.005^{*}$ & .039 & .899 & .059 & .037 & .276 & $.005 *$ & .038 & .899 & $-.093 *$ & .039 & .085 \\
\hline
\end{tabular}

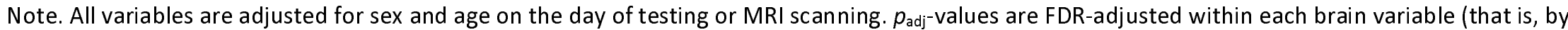
rows of this table). ${ }^{*}=$ significantly (absolutely) lower than the $B$ for PSMD; $\dagger=$ significantly (absolutely) higher than the $B$ for PSMD. Standardised regression coefficients are reported throughout. 
Table 3

The association between structural brain imaging parameters and domains of cognitive ability and general cognitive ability.

\begin{tabular}{|c|c|c|c|c|c|c|c|c|c|c|c|c|c|c|c|}
\hline & \multicolumn{3}{|c|}{ Processing speed } & \multicolumn{3}{|c|}{ Visuospatial ability } & \multicolumn{3}{|c|}{ Verbal memory } & \multicolumn{3}{|c|}{ Crystallised ability } & \multicolumn{3}{|c|}{ General cognitive ability } \\
\hline & 8 & SE & $p_{\text {adi }}$ & 8 & SE & $p_{\text {adj }}$ & 8 & SE & $p_{\text {adj }}$ & 8 & SE & $p_{\text {adj }}$ & 8 & SE & $p_{\text {adj }}$ \\
\hline PSMD & -.273 & .040 & $4.64 \times 10^{-11}$ & -.235 & .042 & $3.66 \times 10^{-08}$ & -.168 & .045 & $2.37 \times 10^{-04}$ & -.072 & .040 & .070 & -.250 & .041 & $2.57 \times 10^{-09}$ \\
\hline General FA & .238 & .046 & $1.07 \times 10^{-06}$ & $.174^{*}$ & .048 & $4.44 \times 10^{-04}$ & .116 & .050 & .021 & .100 & .043 & .021 & .204 & .047 & $2.89 \times 10^{-05}$ \\
\hline General MD & $-.178^{*}$ & .046 & $5.76 \times 10^{-04}$ & $-.163^{*}$ & .047 & .002 & -.117 & .050 & .025 & $.003^{*}$ & .043 & .951 & $-.148^{*}$ & .047 & .003 \\
\hline WMH volume & -.247 & .042 & $1.39 \times 10^{-08}$ & $-.132^{*}$ & .044 & .004 & -.137 & .046 & .004 & -.048 & .040 & .231 & $-.184^{*}$ & .043 & $4.19 \times 10^{-05}$ \\
\hline Grey matter volume & .315 & .039 & $6.42 \times 10^{-16}$ & .310 & .041 & $4.46 \times 10^{-14}$ & .201 & .044 & $3.97 \times 10^{-06}$ & $.253+$ & .037 & $7.12 \times 10^{-12}$ & $.357+$ & .038 & $3.95 \times 10^{-20}$ \\
\hline White matter volume & $.364 t$ & .037 & $3.94 \times 10^{-22}$ & .280 & .042 & $1.64 \times 10^{-11}$ & .175 & .045 & $8.82 \times 10^{-05}$ & $.239+$ & .037 & $1.21 \times 10^{-10}$ & .348 & .038 & $2.45 \times 10^{-19}$ \\
\hline Atrophy & .331 & .039 & $5.89 \times 10^{-22}$ & .277 & .042 & $5.63 \times 10^{-11}$ & .200 & .045 & $1.06 \times 10^{-05}$ & $.169+$ & .038 & $1.33 \times 10^{-05}$ & .320 & .039 & $9.88 \times 10^{-16}$ \\
\hline
\end{tabular}

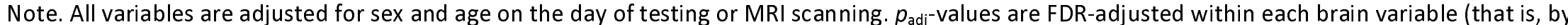
rows of this table). ${ }^{*}=$ significantly (absolutely) lower than the $B$ for PSMD; $\dagger=$ significantly (absolutely) higher than the $B$ for PSMD. Standardised regression coefficients are reported throughout. 
Table 4

Multivariate models predicting latent cognitive factors from all brain variables, entered simultaneously.

\begin{tabular}{|c|c|c|c|c|c|c|c|c|c|c|c|c|c|c|c|}
\hline & \multicolumn{3}{|c|}{ Processing speed } & \multicolumn{3}{|c|}{ Visuospatial ability } & \multicolumn{3}{|c|}{ Verbal memory } & \multicolumn{3}{|c|}{ Crystallised ability } & \multicolumn{3}{|c|}{$\begin{array}{l}\text { General cognitive } \\
\text { ability }\end{array}$} \\
\hline & 8 & SE & $p_{\text {adj }}$ & 8 & SE & $p_{\text {adj }}$ & 8 & SE & $p_{\text {adj }}$ & 8 & SE & $p_{\text {adj }}$ & 8 & SE & $p_{\text {adj }}$ \\
\hline PSMD & 0.093 & 0.060 & 0.240 & -0.184 & 0.063 & 0.024 & -0.095 & 0.068 & 0.416 & -0.043 & 0.058 & 0.525 & -0.146 & 0.060 & 0.032 \\
\hline General FA & -0.010 & 0.051 & 0.969 & -0.066 & 0.055 & 0.365 & -0.045 & 0.058 & 0.534 & 0.037 & 0.050 & 0.525 & -0.026 & 0.052 & 0.813 \\
\hline General MD & 0.002 & 0.052 & 0.969 & -0.053 & 0.055 & 0.447 & -0.043 & 0.059 & 0.534 & 0.067 & 0.051 & 0.470 & -0.008 & 0.052 & 0.880 \\
\hline WMH volume & 0.140 & 0.053 & 0.021 & 0.034 & 0.057 & 0.548 & -0.076 & 0.060 & 0.416 & -0.008 & 0.052 & 0.872 & -0.062 & 0.054 & 0.406 \\
\hline Grey matter volume & -0.035 & 0.059 & 0.788 & 0.173 & 0.062 & 0.024 & 0.099 & 0.069 & 0.416 & 0.125 & 0.057 & 0.112 & 0.149 & 0.059 & 0.032 \\
\hline White matter volume & -0.223 & 0.055 & $1.83 \times 10^{-04}$ & 0.120 & 0.059 & 0.084 & 0.047 & 0.064 & 0.534 & 0.132 & 0.053 & 0.104 & 0.170 & 0.056 & 0.008 \\
\hline Atrophy & -0.220 & 0.048 & $3.69 \times 10^{-05}$ & 0.122 & 0.052 & 0.051 & 0.124 & 0.056 & 0.224 & 0.056 & 0.047 & 0.470 & 0.171 & 0.049 & 0.004 \\
\hline Perivascular spaces & -0.023 & 0.042 & 0.788 & -0.030 & 0.045 & 0.548 & 0.028 & 0.048 & 0.552 & 0.032 & 0.042 & 0.525 & 0.016 & 0.043 & 0.816 \\
\hline$R^{2}$ & & & 0.229 & & & 0.159 & & & 0.082 & & & 0.081 & & & 0.214 \\
\hline
\end{tabular}

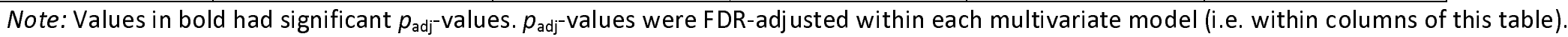

